\title{
ArgumentBind - Um Modelo para Implementação de Aplicações da Argument Web Integradas com Bases de Dados Abertos e Ligados
}

\author{
Alternative Title: ArgumentBind - A Model for Implementing \\ Argument Web Integrated Applications with Open and Linked \\ Data
}

\author{
Roberto Niche \\ Programa de Pós-Graduação em \\ Computação Aplicada \\ PIPCA - Unisinos \\ Av. Unisinos 950, Bairro Cristo Rei, \\ São Leopoldo, RS \\ robertoniche@yahoo.com.br
}

\author{
Sandro J. Rigo \\ Programa de Pós-Graduação em \\ Computação Aplicada \\ PIPCA - Unisinos \\ Av. Unisinos 950, Bairro Cristo Rei, \\ São Leopoldo, RS \\ rigo@unisinos.br
}

\begin{abstract}
RESUMO
As ferramentas de comunicação e colaboração através da Internet são amplamente utilizadas por usuários que, através delas, expressam suas opiniões e descrevem seus pontos de vista sobre os mais diversos assuntos. Entretanto estas ferramentas não foram projetadas para apoiar a identificação precisa dos assuntos tratados e tampouco para permitir o relacionamento de elementos que compõe uma interação, resultando em uma grande disponibilidade de informações mas também uma dificuldade na identificação dos elementos de destaque dessas informações, bem como dos seus relacionamentos e suas fontes. Quando integrada com a iniciativa de bases de dados abertos e ligados, a Argument Web apresenta um potencial de ampliar a qualidade das discussões colaborativas na Web, bem como a sua análise. Neste trabalho é apresentado um modelo para a instanciação deste tipo de aplicações. O diferencial que o modelo apresenta está relacionado com a integração de fontes externas em formatos de bases de dados ligados e com aspectos de visualização de dados. Uma avaliação deste modelo em um estudo de caso com uso de bases de dados abertas e ligadas no âmbito da administração pública é apresentada, indicando boas perspectivas.
\end{abstract}

\section{Palavras-Chave}

Argument Web, Bases de dados abertas e ligadas governamentais, Visualização.

\begin{abstract}
Online communication and collaboration tools are widely employed by users in order to express their opinions on a vast amount of subjects. These tools were not designed to accurately identify topics, nor to point out relationships between topic elements related to a given discussion. As it turns out, there is a staggering amount of user-contributed information generally
\end{abstract}

Permission to make digital or hard copies of all or part of this work for personal or classroom use is granted without fee provided that copies are not made or distributed for profit or commercial advantage and that copies bear this notice and the full citation on the first page. To copy otherwise, or republish, to post on servers or to redistribute to lists, requires prior specific permission and/or a fee.

SBSI 2015, May 26-29, 2015, Goiânia, Goiás, Brazil.

Copyright SBC 2015. available, and conversely, a huge challenge related to accurately pointing out featured topics and their intertwining relationships and sources. The main purpose of Argument Web is to define a rich, full-featured annotation model capable of storing relationships between topics and their sources. Given the availability of rich, interlinked data about a number of related subjects, Argument Web can potentially increase the quality of online discussions and the analysis of its components. However, there is still a reduced number of real-world applications based on these concepts. Even in well-known projects, there's still a lack of exploration efforts related to the use of open and linked data. This paper presents an application model based on Argument Interchange Format (AIF) and state-of-the-art semantic Web technology. Its main contribution lies in the integration of external source information, linked data formats and data visualization aspects. The solution is then evaluated through a case-study related to the use of open and linked data on the field of public administration..

\section{Categories and Subject Descriptors}

Inovação aberta - Dados abertos governamentais - Modelos e processos de participação social - Mídias sociais no governo.

\section{General Terms}

Argument Web, Web Semântica..

\section{Keywords}

Argument Web, Linking Open Data, .

\section{INTRODUÇÃO}

A utilização das ferramentas de comunicação e colaboração através da Internet pode ser considerada como um fato cada vez mais integrado aos hábitos dos seus usuários. Algumas ferramentas, tais como os fóruns de discussão, as redes sociais, os ambientes para blogs e as listas de e-mail, entre outras, são amplamente utilizadas por usuários que, através delas, expressam suas opiniões e descrevem seus pontos de vista sobre os mais diversos assuntos [1]. O conceito de Web 2.0 está associado com este tipo de ferramenta que possibilita um maior interação e protagonismo dos usuários, tendo sido popularizado pela empresa americana O'Reilly Media ${ }^{1}$, com o intuito de destacar a

1 O’REILLY, 2005 
disponibilidade de um nível de serviços diferenciado na Web e atendendo aos seus usuários com diversas ferramentas que estimularam o aumento na geração de conteúdo de forma espontânea e descentralizada.

Uma das utilizações bastante conhecidas das ferramentas da Web 2.0 é o debate sobre assuntos diversos. Uma das ferramentas muito utilizadas para este fim são as redes sociais, como por exemplo a maior rede social do mundo atualmente, o Facebook ${ }^{2}$. Nela é possível verificar a ocorrência de mensagens sobre um determinado assunto, mas não é possível tornar esta mensagem um assunto com possibilidade de originar debates e gerar conclusões efetivas, pois normalmente as mensagens são apenas uma manifestação de opinião. Como exemplo de sua utilização para assuntos diversos, pode ser citado que no final de 2013 a rede social Facebook lançou sua retrospectiva[2] mostrando os assuntos mais comentados no ano, sendo que no caso do Brasil os assuntos mais populares foram: Manifestações populares, Carnaval, Neymar, Rock in Rio, Papa Francisco, Mensalão, Copa das Confederações, Maracanã, Vem pra rua e Boate Kiss. Observa-se uma diversidade de assuntos, inclusive com três deles relacionados à política brasileira, que no caso são: Manifestações, Mensalão e Vem pra rua.

O avanço da Web 2.0 levou ao aumento e diversificação do conteúdo publicado na Web. Entretanto as suas ferramentas não foram projetadas para apoiar a identificação precisa dos assuntos tratados. Tampouco estas ferramentas foram desenvolvidas para permitir o relacionamento de elementos que constituem uma interação para debater um determinado assunto. Por exemplo, em um debate realizado em um fórum de discussões, a identificação dos elementos a favor ou contra uma determinada tomada de posição somente pode ser realizada pela leitura e interpretação por um usuário, sendo que o mesmo se aplica para o caso das validações dos argumentos colocados.

Este contexto descreve a existência de um grande número de meios possibilitando que as pessoas se expressem e gerem conteúdo. Os argumentos utilizados podem ser explorados com o objetivo se obter informações importantes nas mais diversas áreas. Entretanto o formato textual utilizado não permite a sua utilização por ferramentas automáticas de forma efetiva. Além disso o grande volume de dados e sua diversidade podem ser obstáculos para o uso humano. Sendo assim pesquisadores vêm buscando alternativas para estabelecer bases para a World Wide Argument Web (WWAW), que consiste em uma infraestrutura que permita a anotação das mensagens textuais contendo elementos de debates e discussões, de acordo com suas funções no diálogo e com a possibilidade de identificação precisa de suas fontes [3].

Segundo[4], a grande utilização de ferramentas digitais de mediação e colaboração ampliou muito a capacidade de realização de debates sobre os mais diversos assuntos. Entretanto diversos fatores contribuem para que os resultados obtidos em muitas situações sejam insatisfatórios, resultando em debates com poucos argumentos validados e de qualidade, além de uma grande quantidade de mensagens que tende a dificultar a realização de sínteses. Entre os fatores relacionados com esta situação estão a grande quantidade de pessoas que podem enviar mensagens, o contexto de uma grande disponibilidade de informações não validadas e mesmo sem origem, a dificuldade de identificação e relacionamento dos argumentos específicos citados e referidos em diferentes mensagens. A disponibilização crescente dos dados abertos governamentais pode se tornar uma fonte confiável de dados para ser a referencia das postagens e dar credibilidade aos argumentos.

O termo dados ligados (Linked Data), refere-se a um conjunto de melhores práticas para a publicação e interligação de dados estruturados na WEB. Essas melhores práticas foram introduzidas por Tim Berners-Lee em sua nota para a arquitetura Web para dados ligados e tornaram-se conhecidos como os princípios para dados ligados[7]. O propósito central da Argument Web está relacionado com a definição de uma infraestrutura para anotar os argumentos de forma precisa e possibilitar que estes sejam relacionados com suas diversas fontes. Além disso, podem ser desenvolvidas ferramentas para a manipulação e visualização destes dados. Estes estudos, por sua vez, têm estimulado iniciativas nos mais diversos campos de pesquisa, tais como filosofia, comunicação [10], linguística e inteligência artificial [12] e também de áreas comerciais como e-business [12] e egovernment [13].

O desenvolvimento da Argument Web está relacionado com a estruturação da Web Semântica [14], que é uma iniciativa para o desenvolvimento das bases para descrições de conteúdos disponibilizados na Web de modo a facilitar o seu uso automático. Uma das características observadas atualmente como resultado do desenvolvimento da Web Semântica são as bases de dados Abertos e Ligados, que se constituem de conjuntos de dados anotados segundo padrões que permitem a utilização automática destes dados em contextos de processamento automático [7].

Os principais desafios para a implantação da Argument Web estão relacionados com o estabelecimento de uma base conceitual e de um formalismo para a descrição de elementos de debates. Esta necessidade vem sendo atendida pelo surgimento do padrão denominado Argument Interchange Format (AIF) [3], que descreve as fundações para a descrição dos elementos e de seu relacionamento. Além disso, outro desafio está associado com o desenvolvimento de aplicações que possibilitem a interação amigável com os usuários para a anotação de suas mensagens de acordo com este formalismo. Outros fatores importantes estão relacionados com a utilização dos dados anotados por mecanismos de processamento automático que possibilitem a verificação de sua consistência, a sua visualização e o seu resumo, entre outros aspectos. Além disso, a integração de dados disponibilizados a partir dos formatos da Web Semântica, como no caso das iniciativas de dados abertos ligados também é bastante importante para ampliar as possibilidades dos sistemas voltados para Argument Web [5]. Por fim, as metodologias para a avaliação dos sistemas de Argument Web também carecem de realização de pesquisas, devido à sua natureza diferenciada de outros sistemas de interação e colaboração.

Este trabalho apresenta um modelo que possibilita o encaminhamento de algumas destas demandas, fomentando a implementação de aplicações de Argument Web. O modelo busca atender às necessidades de um ciclo que inicia com a interação dos usuários para a descrição e anotação das mensagens; segue com operações de visualização dos resultados gerados; permite a integração de fontes para corroboração dos argumentos a partir de bases de dados ligados disponíveis na internet; por fim, descreve os dados gerados no formato AIF para possibilitar a sua manipulação de forma automatizada.

O diferencial que o modelo apresenta está relacionado com a integração de fontes externas em formatos de bases de dados abertos e/ou ligados, o que possibilita que os usuários dos 
sistemas descritos com este modelo possam se valer de recursos existentes na Internet para associar às suas mensagens, desta forma apoiando a validações de seus argumentos.

A expressão governo aberto refere-se a projetos e ações que visam à promoção da transparência, à luta contra a corrupção, ao incremento da participação social e ao desenvolvimento de novas tecnologias, de modo a tornar os governos mais abertos, efetivos e responsáveis3. Em destaque encontra-se Estados Unidos da America que teve seu início em 05/2009 e na sequencia Reino Unido em 09/2009, Nova Zelândia em 11/2009. O Brasil assinou a Declaração de Governo Aberto em 11/2011[9], quando ocorre o lançamento da parceria do Governo Aberto ou OGP4. Os países signatários da Declaração de Governo Aberto reconhecem a exigência por governos mais transparentes, bem como as demandas sociais por maior participação popular nos temas públicos. Podendo assim, utilizar destas informações para desenvolvimento de ferramentas, modelos e processos de participação social dos governos, buscando maior interação com seu público e eleitores.

Acredita-se que este tipo de iniciativa de integração dos recursos de bases de dados abertas e ligadas governamentais, juntamente com aplicações de Argument Web é positivo considerando o contexto atual em que existem diversas formas para que os usuários da Internet realizem manifestações, porém com dificuldade de validação ou evidenciação da validade das conclusões e argumentos apresentados. Esta abordagem também atua no sentido de promover a concretização das ideias de inteligência coletiva, tal como descritas por Pierre Levy[15]. Dada a grande quantidade de postagens sobre os mais diversos assuntos e problemas, é necessária a utilização de um conjunto de ferramentas capaz de poder apoiar este momento vivido pela sociedade, facilitando a gestão dessas informações a ponto de disponibilizar uma estrutura em que a população seja capaz de efetuar suas conclusões críticas e fidedignas sobre os debates existentes.

O restante do texto está organizado como segue. Na seção 2 são apresentados elementos de trabalhos relacionados que subsidiaram as escolhas do modelo proposto, que é descrito na seção 3. A seção 4 apresenta detalhes do estudo de caso e avaliação preliminar. A seção 5 apresenta as conclusões do artigo.

\section{TRABALHOS RELACIONADOS}

Nesta seção são descritos e analisados brevemente trabalhos relacionados com a proposta apresentada.

O trabalho de [3] cita que nos últimos anos, um número expressivo linguagens de marcação de argumentos vem sendo usado no desenvolvimento de ferramentas de visualização e discussão de argumentos. Um exemplo é a Assurance and Safety Case Enviroment (ASCE), cuja ferramenta foi desenvolvida para efetuar a gestão de casos de garantia, segurança e documentações de projetos complexos. Esta ferramenta é baseada em uma ontologia sobre segurança com base em reivindicações, argumentos e provas. Outra citação do autor é a análise realizada nos estudos de argumentação humana, que levou ao desenvolvimento de outras linguagens de marcação de

3 http://governoaberto.cgu.gov.br

4 Open Government Partnership argumentos especializados. Segundo o autor, dois sistemas de maior expressão neste sentido são ClaiMaker [17] e AML [18].

Estas diversas tentativas de desenvolver uma linguagem de marcação de argumentos compartilhados tem duas grandes limitações. O primeiro problema é que essas linguagens são utilizadas em ferramentas específicas. O segundo problema está na marcação, que compreende que o usuário terá que estruturar seus argumentos através de diagrama de sentenças em linguagem natural. Assim, estas linguagens de marcação não são projetadas para processar instruções lógicas formais, como aqueles utilizados em sistemas multi agentes.

Para resolver essas questões um grupo de pesquisadores em argumento e computação reuniram-se para um workshop onde o objetivo foi esboçar um formato de argumentação intercambiável "Argumentation Interchange Format (AIF)" que consolida sempre que possível com os trabalhos já existentes de linguagem de marcação e frameworks de sistemas multi-agentes. Os principais objetivos da AIF foram: a) facilitar o desenvolvimento de sistemas multi-agentes (fechados ou abertos), que seja capazes de usar raciocínio baseado em argumentação e interação utilizando um formalismo compartilhado; b) facilitar o intercâmbio de dados entre as ferramentas para manipulação e visualização de argumentos.

No decorrer deste trabalho o autor demonstra uma visão geral do modelo proposto pelo AIF, sua aplicação e as conclusões. A figura 1 representa este modelo, e pode-se observar que existem dois tipos de elementos "node" no cerne da AIF, o node de informação (ou I-node) que mantém as informações ou dados, e o node de plano (ou $S$-node) que representa a transição de inferência associado com uma declaração argumentativa. Os nodes $S$-node tem a função de capturar as aplicações do esquema, ou seja, os padrões de raciocínio. Este sistema pode ter padrões independentes de domínio de raciocínio, semelhante ao uso das regras de inferência em lógica dedutiva, mas que podem ser ampliadas para lógicas não dedutivas. Na figura 1 é possível observar alguns desses padrões, como por exemplo regras de aplicação de inferência (RA-NODE), conflitos de aplicação(CANODE), preferências de aplicação (PA-NODE), e assim podem-se

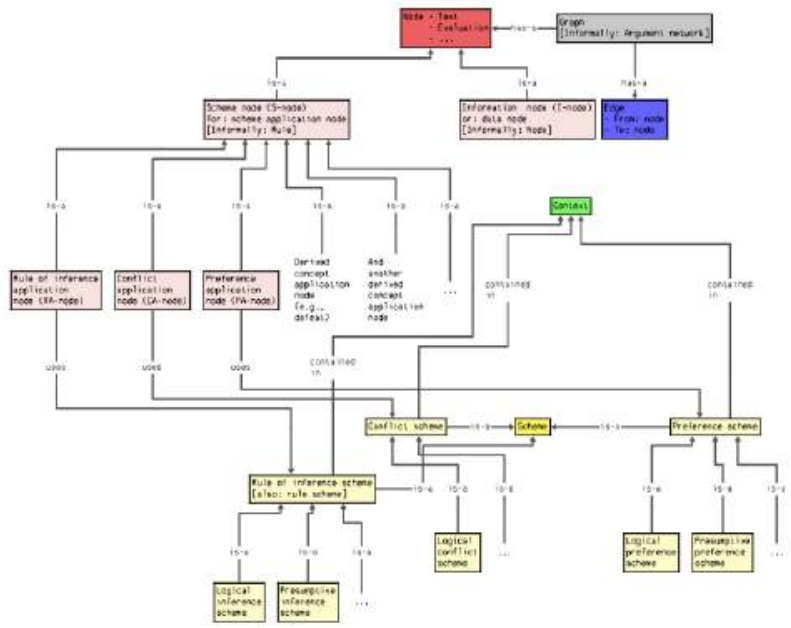

criar outros tantos quanto necessários. Finalmente, as propriedades e as relações entre classes e instâncias (incluindo arestas (EDGE) do grafo(GRAPH)) serão capturados através de predicados sobre os conjuntos. 


\section{Figura 1:Modelo conceitual e relacional para uma ontologia de argumentos}

O trabalho descreve a realização de alguns testes com modelos abstratos e posteriormente o autor conclui que no modelo de testes, apesar de abstrato, requer diferentes características de áreas cientificas (argumentação teórica, sistemas multiagentes, lógica não clássica). Neste contexto ele observa que a proposta do modelo abstrato surgiu de um trabalho conjunto entre pesquisadores das diversas áreas cientificas tentando abranger todas as áreas, porém, é ressaltado que este modelo pode não capturar todos os tipos de argumentação de interesse, sendo assim, algumas questões significativas surgiram durantes as discussões que são:

- Utilizar o formalismo AIF com ferramentas de importação-exportação que possa ser utilizado em comunicação entre agentes. Enquanto os conceitos fundamentais podem ser os mesmos, continua a ser uma questão em aberto saber se um formato realmente pode cobrir adequadamente ambos os casos.

- Dada a riqueza potencial da ontologia os conceitos de comunicação continuam a ser uma questão em aberto como as Agent Communication Languages (ACL), podem ser utilizadas, sendo que outra preocupação é quanto ao seu reuso.

- Como deve ser a organização da comunidade de usuários ao redor da AIF, de acordo sobre conceitos fundamentais e as extensões?

No trabalho de [16] o autor ressalta a proposta demonstrada no artigo "Towards an Argument Interchange Format" e propõe construir sobre os pontos fortes e as potencialidades da Web Semântica, uma ontologia utilizando a linguagem de ontologias da Web Semântica e o RDF Schema. O autor apresenta um projeto piloto no qual ele denomina ArgDF. Neste projeto os usuários podem criar seus argumentos de diferentes "Argumentation schemes" e podem também fazer consultas usando a "Semantic Web Query Language".

ArgDF também oferece funcionalidades flexíveis para criação de novos "Argumentation schemes" a partir da interface do usuário. Sendo assim, o ArgDF é uma plataforma aberta não somente para representação de argumentos, mas também para unir "interlinks" e uma rede de argumentos dinâmicos na Web Semântica. Esta iniciativa é para uma ferramenta de domínio público que tem a intenção de propagar um rico conjunto de aplicativos sofisticados para criação, links, navegação, busca e evolução da Argument Web.

Este artigo estuda a modelagem computacional da argumentação em três linhas. Primeiro, são apresentadas as anotações, navegações e manipulações da "Semantic Web-based". Em segundo é demonstrado a primeira representação altamente escalável e fortemente estruturada a capacidade da representação dos argumentos para a Web. Finalizando o trabalho, o autor contribui para a proposta "Argument Interchange Format (AIF) ontology"[3], estendendo-a para capturar os esquemas de argumentos de Walton [18] e fornecendo uma completa implementação do padrão AIF. O autor cita que se for bem sucedido este será o maior sistema de apoio a argumetação já construído, pois sua construção não é centralizada mas distribuída através de contribuintes e desenvolvedores de softwares. $\mathrm{O}$ autor conclui que o papel da AIF e sua implementação se tornará cada vez mais importante conforme os números de argumentações na $W e b$ crescem em sofisticação, número e popularidade.
O uso de ferramentas de publicação conhecidas como BLOGS tornou-se comum na comunidade on-line, como sendo a adoção de uma plataforma aberta para compartilhar e discutir informações e opiniões [6]. Atualmente existem quase 60 milhões de blogs no Tumblr e Blogspot/Blogger classifica consistentemente como um dos sites mais visitados na Web. Muitas das ferramentas existentes para a interface com a Argument Web são voltadas para o público especializado (usuários acadêmicos interessados no discurso e análise de argumento). O mesmo é verdadeiro para um grande número de ferramentas discutidas em [20].

O ArguBlogging possui como objetivo abrir a Argument Web aos internautas regulares com uma visão crítica: os blogueiros. Uma característica que o autor informa é que sua ferramenta não requer instalação e faz uso do bookmarklet. Com os recursos de bookmarklet os usuários podem emitir suas opiniões de determinado texto em alguma página apenas destacando algum termo e após isso clicando no bookmarklet do ArguBlogging. Assim o ArguBlogging é processado na página dispondo de opções para respostas.

As pessoas podem ir navegando por sites da $W e b$ e assim que visualizar um termo, uma notícia, ou qualquer informação sobre a qual queiram emitir sua opinião, basta o usuário destacar o termo e clicar no bookmarklet do ArguBlogging para a ferramenta ser processada e abrir a caixa de diálogo com as opções para indicar que concorda ou discorda, mais a caixa de texto para emissão da opinião, selecionar qual o blogger em que deseja salvar sua opinião e clicar no botão de enviar. $\mathrm{O}$ autor conclui que ao fornecer um bookmarklet com uma interface simples e fácil de usar, dados estruturados e semanticamente ricos podem ser coletados dos usuários de uma forma discreta. Desta forma, o autor pretende incentivar o crescimento direto da Argument Web e da Web Semântica. As avaliações preliminares sugerem que esta abordagem para coleta de metadados é atraente e o feedback dos usuários é particularmente apropriado para estendê-lo para aplicações da Argument Web para Twitter e Facebook.

$\mathrm{O}$ autor comenta que o ArguBlogging foi construído como um alicerce para blogs semânticos [21]. Assim, a argumentação de conexão e ontologias para as mídias sociais, que já foi explorado em [22], pode ser feita facilmente. A conexão entre blogs e dados vinculados é semanticamente rica e abre novas possibilidades de pesquisa em áreas como a mineração de opinião e análise de sentimento, por exemplo.

Atualmente, a ferramenta ArguBlogging permite apenas o discurso argumentativo muito simples: dada uma reclamação, pode-se concordar ou discordar. A pesquisa atual está trabalhando em processamento generalizado de sistemas de diálogo formais [23], que fornecem regras para o diálogo coerente. Em [5], o autor propõe um quadro genérico para capturar esses sistemas de diálogo, o que é explicitamente compatível com a ontologia AIF. Sendo assim, este modelo permite uma maior variedade de movimentos argumentativos. Por outro lado, isso torna ArguBlogging não apenas um sistema para descrever ou capturar discurso argumentativo on-line, mas também para melhorar tal discurso. As regras de um sistema de diálogo, por exemplo, podem explicitamente proibir ataques contra a pessoa, deixando que o debate foque no que realmente está em jogo. Assim o ArguBlogging deve ajudar a promover o objetivo normativo da Argument Web, que é trazer um novo tipo de racionalidade crítica para a Web.

Portanto, pode-se verificar que atualmente o número de trabalhos utilizando o suporte para a Argument Web ainda é 
pequeno, sendo que os trabalhos existentes não se preocupam com a integração mais ampla de bases de dados abertas e ligadas. Por outro lado, considera-se que pode ser uma vantagem relevante a utilização destes sistemas em conjunto com os dados disponibilizados pelas iniciativas de governo eletrônico e de dados abertos conhecidas. Também nota-se a carência por aplicações e modelos para a criação de aplicações que utilizem os preceitos da Argument Web, além do aproveitamento das bases de dados abertas e ligadas governamentais.

\section{MODELO PROPOSTO}

O objetivo do modelo proposto é apoiar a ampliação do uso de bases de dados ligadas e abertas, além das iniciativas de governo eletrônico, proporcionando a construção de aplicações de Argument Web para proporcionar o debate entre usuários e manter este debate aberto com argumentos justificáveis e que, se possível, contenham relações com alguma base de dados aberta e ligada, causando um impacto maior na credibilidade dos argumentos publicados. A seguir são descritos os principais elementos desta arquitetura.

\subsection{Arquitetura geral}

O modelo conta com um sistema de seleção de assuntos para proporcionar uma base de filtro para busca de dados ligados. De acordo com o assunto dos debates e os itens relacionados, o modelo deverá ter condições de buscar informações na Web sobre o que o usuário estiver argumentando para dar credibilidade a sua postagem. A busca na Web ocorrerá através de uma base de dados que será indicada previamente de acordo com os assuntos tratados.

$\mathrm{Na}$ figura 2 é possível ter uma visão da arquitetura desenvolvida, com os principais componentes. Os componentes de cadastro e controle de acesso serão responsáveis por gerir os dados dos usuários bem como suas permissões para acessos ao banco de dados interno e o banco de dados AIF. O componente de reputação possui como objetivo manter dados sobre a reputação dos usuários cadastrados no sistema e o seu histórico de interações.
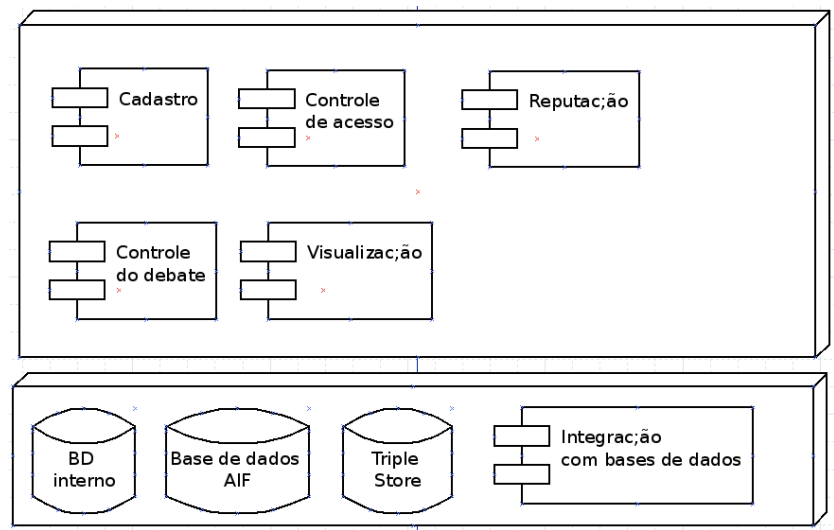

Figura 2. Arquitetura geral

Já o componente de controle do debate é responsável por gerir o debate, em atividades como criar o debate, enviar as mensagens e respostas, exibir a classificação das respostas e efetuar a relação com as fontes de dados ligadas. No componente de visualização será possível obter uma visão gráfica do debate. Um exemplo é a visualização em linha de tempo, indicando o rumo que o debate tomou em terminado período. Outra visualização que será possível é o formato de aglomeração, onde as mensagens com a mesma classificação tendem a ficar mais próximas.

O componente de integração com bases de dados será o responsável por efetuar a manutenção das fontes externas do sistema. Algumas possíveis fontes de dados externas são aquelas já disponibilizadas com o formato de bases de dados abertas e ligadas, cujo conteúdo pode ser utilizado para popular o armazenamento em bases triple-store. Já as ferramentas de coleta e organização de dados (por exemplo, a ferramenta Pipes do Yahoo, ou a ferramenta Deri) também podem ser utilizadas, a partir de configurações de formatos específicos.

\subsection{Principais etapas do processo}

Entre as principais estão o cadastro do usuário, as postagens e a manutenção do banco de dados abertos e/ou ligados. Tanto o cadastro quanto a postagem seguem o padrão utilizado pelos sistemas de debates, sendo importante destacar a manutenção do banco dados aberto e/ou ligados, como é possível analisar na figura 3 .

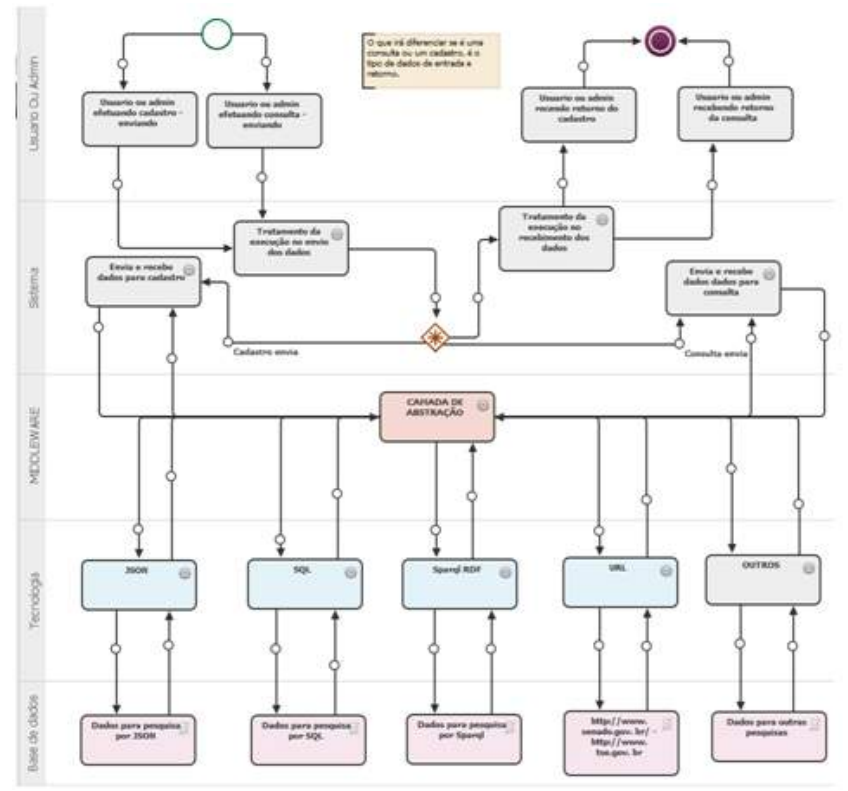

Figura 3. Modelo abstrato de consulta e cadastro das bases de dados ligados

A pesquisa por dados ligados deverá ocorrer por diversos meios. Na figura 3 a camada sistema entende-se como o usuário pesquisando ou até mesmo o administrador do sistema fazendo o cadastro. Logo tem-se que o processo deve ser semelhante, onde o sistema irá fazer uma pesquisa em alguma base pré-cadastrada na camada da base de dados. A camada de tecnologia deverá ter o processo automatizado, já preparado para receber diversas formas de consultas, porém cada uma destas formas terá uma programação específica pois se tratam de diversas tecnologias. A camada de middleware representa a interface que tanto o administrador quanto os usuários terão acesso, logo entende-se que tanto para efetuar uma consulta, quando para efetuar o cadastro de uma base de dados ligados, o sistema deverá ter o mesmo comportamento, nesta camada que será tratada a pesquisa e cadastro para que o modelo tenha maior eficiência na sugestão de fontes de bases ligadas bem como maior facilidade de cadastramento de informações. 


\subsection{Arquitetura de informação e Protótipos \\ de telas}

O modelo prevê 5 telas essenciais: a primeira tela é onde tudo inicia, um formato padrão de sistemas de debates, onde o usuário efetua seu cadastro e/ou navega pelo sistema com o objetivo apenas de ler as postagens dos debates. A segunda tela importante é a formação do debate, onde o usuário irá escolher o assunto, o nível do assunto, podendo ser este assunto desde muito genérico até muito especifico. A terceira tela importante é onde ele visualiza o debate, ou se tiver permissão poderá criar um novo debate conforme o assunto selecionado. A quarta tela importante do sistema é a janela modal, onde o usuário irá postar a sua argumentação sobre o assunto em questão, como pode-se observar na figura 4 .

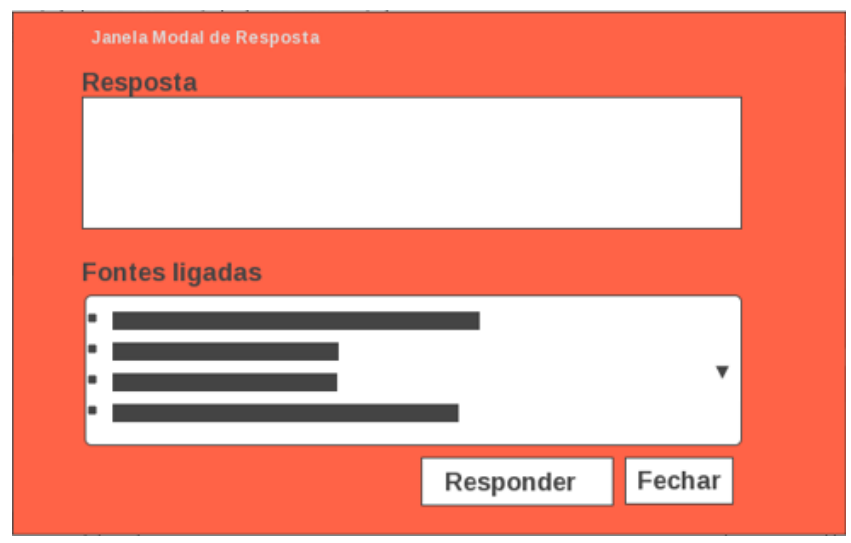

Figura 4. Janela modal

A janela modal é acionada sempre que um usuário desejar argumentar sobre alguma postagem, a partir de botões com a seguintes classificações: concordo/discordo parcialmente, concordo/discordo plenamente. Na figura 4 pode-se observar dois campos, uma a resposta onde o usuário irá efetuar sua argumentação, outro com o campo Fontes Ligadas, que irá conter as fontes que tem relação com o assunto escolhido e também tenham alguma relação com o que o usuário argumentou. Este processo será realizado através da camada middleware, segundo o modelo descrito.

A quinta tela importante contêm as possíveis visualizações do debate. Neste momento o usuário poderá visualizar como o debate transcorreu ao longo do tempo, tendo uma visão por aglomerações, temporal e grau de importância. O modelo da figura 5 proporciona uma visão temporal das postagens. Assim o usuário poderá ter uma visão sobre as postagens em uma determinada data e o rumo que elas foram tomando conforme o período, inclusive poderá ver o que as fontes de dados ligados indicavam em um determinado período que levou o usuário a efetuar a postagem.

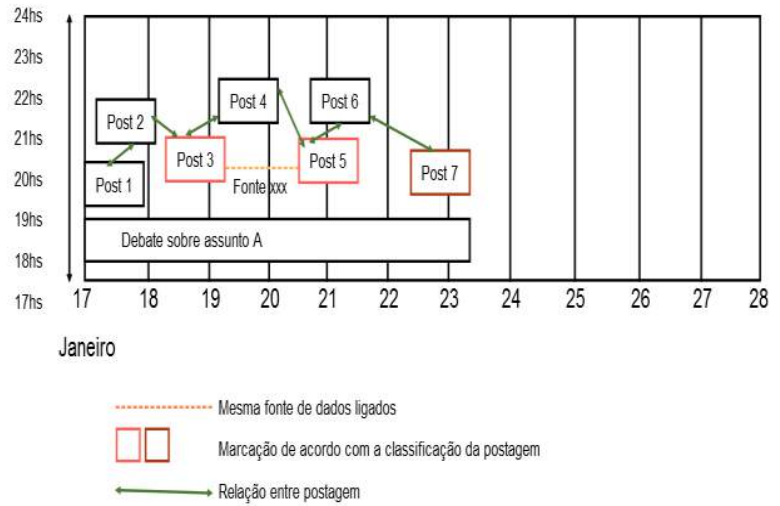

Figura 5. Visualização de postagem por linha de tempo

Conforme a característica observada na figura 6, este também possibilita identificar as postagens conforme sua classificação e seus atributos. Também será disponibilizada uma visão macro das postagens. Esta visão proporciona a noção do grau de importância por assunto de acordo com o período. O grau de importância será avaliado de acordo com a quantidade total de postagens mais a quantidade total de fontes utilizadas mais a quantidade total de apontamentos realizados. Estes apontamentos são as marcações de concordo, discordo e outras sem qualquer argumentação. Sendo assim, quanto maior a interação dos usuários em determinado assunto, mais importante o assunto torna-se.

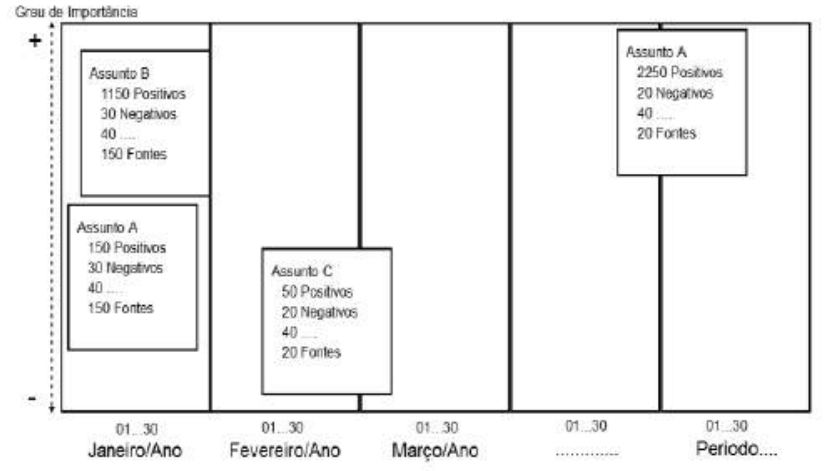

Figura 6. Visualização dos assuntos por ordem de grandeza

\section{Estudo de caso proposto e avaliação}

Para permiti-lo a avaliação do desenvolvimento deste trabalho e aprofundamento sobre a WWAW, foi realizada uma implementação de um sistema de discussão com foco em temas políticos, como por exemplo, saúde, educação, transporte entre outros, sempre relacionados com um político e a sua cidade/município ou estado.

Neste sistema as pessoas podem manifestar suas opiniões sobre seu tema de preferência gerando debates sobre algum assunto em alguma localidade, relacionando com o(s) político(s) em exercícios de acordo com o ano selecionado. Cada postagem realizada pode ser relacionada com outras mensagens formando uma rede de informações com um único objetivo, que seria o de gerar debates e conclusões sobre a situação atual do assunto em questão. Os assuntos foram restritos ao âmbito político governamental brasileiro levando em conta da Lei 12.527, de 18 
de novembro de 2011, que define e orienta sobre a transparência e acesso a dados abertos do governo, de modo a potencializar a utilização das informações atualmente disponíveis, bem como estudar melhorias nas aplicações desta área, buscando uma melhor utilização futura, com base na expectativa de aumento da quantidade de informações governamentais abertas e ligadas.

Já sobre os assuntos que serão debatidos também serão delimitados aos temas como saúde, segurança, transporte, corrupção, inflação, educação e outros. Para cada um dos grandes assuntos o usuário poderá sugerir outros subtemas para debater, ou seja, aprofundar o debate especificando o assunto. Em todo e qualquer assunto o subtema deverá estar ligado a um ou mais político de acordo com a esfera política. Este relacionamento é importante na visão avaliação do sistema, em especial por proporcionar o uso de bases de dados ligadas contendo estas informações.

Segue uma breve descrição dos dados disponibilizados sobre candidatos e políticos. A estrutura definida neste estudo de caso é semelhante à estrutura fornecida pelo $\mathrm{TSE}^{5}$. A população desta estrutura será realizada de duas formas: $1^{\mathrm{a}}$ importando os dados do site do tribunal, utilizando-se da lei de acesso a informação, como parte da arquitetura dos dados ligados abertos. É importante destacar neste momento, que na importação depende da estrutura de dados para determinado tipo de eleição. Por exemplo, eleições para governador e para prefeituras contêm dados diferentes. $\mathrm{O}$ TSE cria um identificador para o candidato e isto em um determinado momento pode passar despercebido levando a acreditar que este identificador irá se repetir nas demais eleições, quando isso não é verdadeiro. A estrutura de dados que é fornecida pelo TSE não vem normalizada na $3^{\text {a }}$ forma normal, logo, foi previsto um módulo no sistema para fazer essa validação para que a importação dos dados seja mais eficiente e rápida dentro da estrutura proposta como pode ser visualizado na figura 7, o processo de transmissão dos dados do TSE para o banco de dados do modelo.

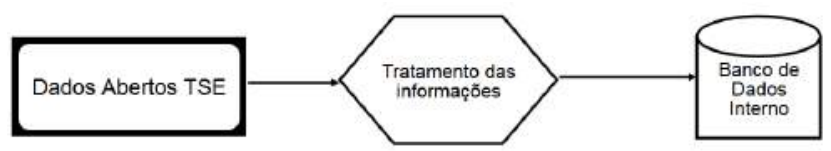

Figura 7. Transmissão dos dados do TSE

$\mathrm{Na}$ figura 8 é possível visualizar uma das telas implementadas do sistema, com dois debate ocorrendo.

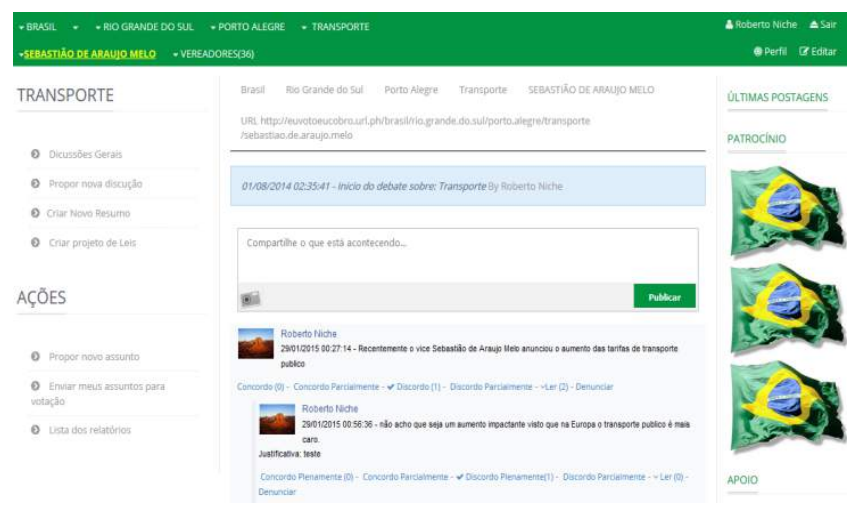

Figura 8. Tela Debate
Pode-se ressalvar na figura 8 , na barra verde os menus são relacionados com opções sobre os dados do âmbito político, e nestes menus são listadas as opções ligadas aos dados abertos, retirados da base do Tribunal Superior Eleitoral. Outro destaque que podemos observar nesta figura é o debate ocorrendo, e as opções que os usuários tem para interagir, que são, concordo/discordo plenamente, concordo/discordo parcialmente e denunciar, o elemento "Ler", ao clicar, irá mostrar todas as postagens filho sobre o debate.

Para a utilização do estudo de caso proposto como elemento da avaliação do modelo foram previstas e parcialmente realizadas as seguintes componentes metodológicas:

a) Testes funcionais: o protótipo implementado neste estudo de caso deve passar por testes funcionais para identificar o correto funcionamento do sistema para todos os seus componentes. Isso inclui desde os componentes voltados para a manutenção de sua infraestrutura, tal como o cadastro de usuários, assuntos e os controles de acesso, bem como as opções de edição e publicação de mensagens e sua anotação, até o uso de bases de dados ligados e a visualização dos resultados das mensagens e debates.

b) Avaliação de usabilidade do sistema, a partir de ensaios de interação com grupos reduzidos de usuários, a serem definidos por conveniência. Nestes testes de interação serão identificados os impactos da estrutura proposta, tal como percebidos pelos usuários nas interações de uso do sistema. Estes elementos serão importantes para eventuais ajustes do sistema prevendo o seu uso na etapa de testes em larga escala.

c) Utilização em larga escala, convidando um número maior de usuários para a utilização livre do protótipo, de modo a possibilitar a observação dos resultados de seu uso e coletar, através de questionários específicos, a impressão e sugestões dos usuários.

Atualmente o sistema foi avaliado parcialmente com base nos itens "a" e "b", como forma de identificar e corrigir necessidades relevantes. As etapas de avaliação de usabilidade e de disponibilização do sistema para uso em larga escala estão previstas para execução em breve.

\section{Conclusões}

Este documento apresenta uma proposta que busca contribuir com a área da Argument Web. No decorrer deste trabalho, observou-se a importância dos sistemas atuais para debate como fóruns e blogs, entre outras. Bem como observaram-se as suas limitações para que a população ativa possa expor seus argumentos, e o debate fluir com coerência e em alto nível. Neste sentido foram estudados os trabalhos relacionados e o que está sendo proposto para solucionar este problema, onde pode-se destacar a ontologia AIF e o sistema ArguBlogging. Ao estudar os trabalhos relacionados foi identificado que a área Argument Web é uma área nova com grandes possibilidades de fomentar aspectos de inovação em participação social e na utilização de bases de dados ligadas e abertas governamentais. Com este estudo não foi identificado um sistema que seja realmente capaz de suprir o problema apontado para os debates, sendo assim, conclui-se que há a necessidade um modelo para atender esta demanda, o que motivou este trabalho desenvolvendo um modelo de estrutura de debates com fontes ligadas e visualizações dos debates.

Destacam-se como contribuições do trabalho o estudo da área e a sua divulgação, a definição de um modelo que pode gerar 
resultados práticos relevantes no âmbito do uso mais produtivo das ferramentas de geração de conteúdo e colaboração na Web, bem como a experimentação no uso pioneiro de bases de dados ligadas e opções diferenciadas de visualização integradas ao contexto da Argument Web.

Um protótipo para o uso deste modelo com base no contexto da discussão de temas ligados à política pública brasileira foi desenvolvido e está sendo progressivamente testado e avaliado dentro do escopo de avaliação funcional, testes de usabilidade e avaliações do uso em larga escala. No momento os testes funcionais, que são de grande relevância para continuação deste trabalho, foram parcialmente realizados, juntamente com os testes de usabilidade e como conclusão observou-se que esta etapa encontra-se em fase final de desenvolvimento, sendo identificados aspectos positivos até o momento.

A sequência e continuidade deste trabalho, com o intuito de obter resultados satisfatórios, envolvem a continuidade desenvolvimento e aperfeiçoamento do modelo proposto em conjunto com o estudo de caso, a realização de novas baterias dos testes previstos e por fim realizar as avaliações críticas e eventuais correções cujas necessidades sejam identificadas.

\section{Bibliografia}

[1] Atkinson, K.; Bench-Capon, T.; Mcburney, Parmenides P.: Facilitating deliberation in democracies. Artificial Intelligence and Law, 2006. retirado do artigo Laying the foundations for a World Wide Argument Web.

[2] Facebook, 11 Setembro 2014. Disponivel em: <http://www.facebookstories.com/2013/pt-br>. Acesso em: 11 Setembro 2014.

[3] Chesñevar, C. et al. Towards an Argument Interchange Format. The Knowledge Engineering Review, Vol. 00:0, p. 1-25, 2007.

[4] Bex, F. et al. Implementing the Argument Web. Communications of the ACM, v. 56, n. 10, p. 66-73, Outubro 2013.

[5] Bex, F.; Reed, C. Dialogue templates for automatic Argument processing. Frontiers in Artificial Intelligence and Applications, vol. 245, p. 266-377, 2012.

[6] Bexa, F. et al. ArguBlogging: An application for the Argument Web. Web Semantics: Science, Services and Agents, p. 9-15, 2014.

[7] Bizer, C. Linked Data Evolving the Web into a Global Data Spac, p. 7-25, 2011.

[8] Cayzer, S. Semantic blogging and decentralized knowledge management. ACM, p. 47-52, 2004.

[9] Governo Aberto, Disponivel em: $<$ http://governoaberto.cgu.gov.br/aogp/o_que_e_Governo_Aberto.asp>. Acesso em: 28 Outubro 2014.

[10] Rahwan, I.; Guillermo, S. Argumentation in Artificial Intelligence. Springer, 2009.

[11] Mark, A.; Miriam, B. G. Argument reconstruction and socio-technical facilitation of large scale
Argumentation. In Proceedings of the 3rd International Conference on the Pragmatic Web: Innovating the Interactive Society, p. 77-81, 2008.

[12] Klein, M. Achieving collective intelligence via large-scale on-line Argumentation. MIT Sloan School of Management, 2007.

[13] Macintosh, A.; Thomas, G. F.; Alastair, R. Providing Argument support for e-participation. Journal of Information Technology \& Politics, p. 6(1):43-59, 2009.

[14] Berners-Lee, T.; Hendler, J.; Lassilia, O. The semantic Web. Scientific American, p. 34-44, 2001.

[15] Lévy, P. Inteligência coletiva: Para uma antropologia do ciberespaço. Loyola, n. 5, p. 212, 2007.

[16] Iyad Rahwan, Fouad Zablith, Chris Reed, Laying the foundations for a World Wide Argument Web, Artificial Intelligence, Volume 171, Issues 1015, JulyOctober 2007, Pages 897-921, ISSN 0004-3702;

[17] Shum, S. B. Sensemaking on the Pragmatic Web: A Hypermedia Discourse Perspective. International Conference on the Pragmatic Web, Stuttgart, p. 06-16, 2006.

[18] Reed, C.; Rowe, G. Araucaria: Software for Puzzles in Argument, 2001.

[19] Walton, D. N. Argumentation Schemes for Presumptive Reasoning, 1996.

[20] Schneider, J.; Groza, T.; Passant, A. A review of Argumentation for the social semantic Web. Semantic Web, 2011.

[21] Cayzer, S. Semantic blogging and decentralized knowledge management. ACM, p. 47-52, 2004.

[22] Lange, C. et al. Expressing Argumentative discussions in social media sites. Social Data on the Web.

Mcburney, P.; Parsons, S. Dialogue Games for Agent Argumentation. Argumentation in Artificial Intelligence, Springer, p. 26 